\title{
Reaction time to the offset of brief auditory stimuli
}

\author{
CHARLES E. ROBINSON* \\ Neurophysiology-Biophysics Research Laboratories \\ Veterans Administration Hospital, Martinez, California 94553
}

\begin{abstract}
An earlier investigator has found that the time at which the offset of a stimulus is perceived depends on the time of stimulus onset, rather than on the time of stimulus offset, for stimuli briefer than a critical duration of the order of $100 \mathrm{msec}$. In an attempt to determine whether this effect extends to reaction time (RT), we have determined RT to the offset of brief noise bursts. RT was found to be essentially constant as a function of stimulus duration. Thus, RT to stimulus offset appears not to be related to the time of perception of offet, as determined in the earlier investigations.
\end{abstract}

The time at which the offset of a brief stimulus is perceived does not depend on the actual time of stimulus offset; rather, for a given set of stimulus parameters, the perception of offset occurs at a fixed interval of time after the stimulus onset. This surprising result has been demonstrated with a remarkable degree of precision in the auditory and visual modalities by Efron (1970a, b, c), and with a lesser degree of precision in the vibrotactile modality by Efron (1973), for stimuli briefer than a critical duration of the order of $100 \mathrm{msec}$. In particular, for the conditions that Efron employed in determining perception of offset in the auditory modality, he obtained a critical duration of $130 \mathrm{msec}$. (Critical duration is defined here as the limiting duration above which perception of offset occurs at a fixed interval of time after stimulus offset.) Efron determined (to within an additive constant) the time at which offset is perceived by requiring Ss to judge whether or not the offset of the test stimulus appeared to be simultaneous with the occurrence of another stimulus.

Efron's unexpected finding leads one to wonder how quantities other than the time of perception of offset might be related to the time of stimulus offset. This question has been explored on the neurophysiological level in cats by Efron (1973). The general finding at a number of locations in the nervous system is that features of evoked potentials which would ordinarily be identified as "off responses" do occur at a fixed time after the stimulus onset, for stimuli briefer than a critical duration on the order of $50 \mathrm{msec}$. The neurophysiological result thus seems to correspond to the result for the time of perception of offset.

Considering again the behavioral level, another quantity associated with stimulus offset is reaction time (RT) to stimulus offset. If the finding for time of perception of offset were to extend to RT, then the

*I wish to thank Mary Corder for collecting and analyzing the data. Present address: Fluid Dynamics Research Department, General Motors Research Laboratories. Warren. Michigan 48090. following equation would hold for brief stimuli:

$$
\mathrm{RT}=\text { constant }-\mathrm{D}
$$

where $D$ is the stimulus duration. This result would be expected if the response to a stimulus offset could be initiated only at a time which closely corresponded to the time of perception of offset. In contrast, the simplest result which might be expected if Efron's result does not extend to RT would be simply

$$
\mathrm{RT}=\text { constant }
$$

In the present paper, we determine RT to the offset of brief noise bursts in an attempt to discover whether or not the result for the time of perception of offset extends to the RT task.

\section{METHOD}

\section{Subjects}

The Ss were two paid undergraduate students, both of whom have clinically normal hearing. They were chosen from a group of eight Ss who were tested earlier in a different RT experiment. The two Ss were chosen on the basis of their performance in the earlier experiments, the criteria being small variability in RTs, both between sessions and within single blocks of trials. The two Ss were given a number of practice sessions on the present experiment.

\section{Apparatus and Stimuli}

Noise bursts were produced by a noise generator (General Radio 1390-B), rectangularly gated by an electronic switch (Siliconix 0137), and filtered by a bandpass filter (Krohn-Hite $3500 \mathrm{R}$ ) set to $500-5,000 \mathrm{~Hz}$. The stimuli were presented to the left ear via Koss PRO-4A earphones. The noise bursts were at an overall SPL of $70 \mathrm{~dB}$ re $0.0002 \mathrm{dyne} / \mathrm{cm}^{2}$.

A normally open telegraph key was used for the response. The

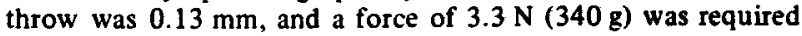
to make contact.

\section{Procedure}

The Ss were asked to respond to the offset of brief noise 


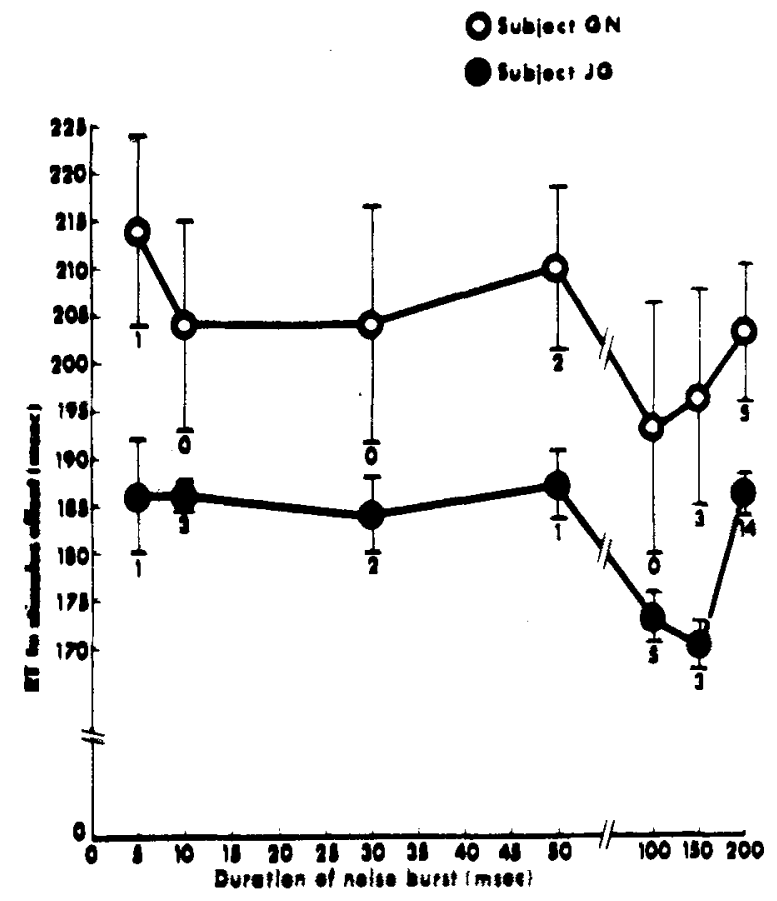

Fig. 1. Reaction time to stimulus offset as a function of stimulus duration. Each point represents the mean of the four corrected mean RTs obtained in four sessions. The error bars indicate \pm 1 standard error of the mean of the four corrected mean RTs. The number of false presses (pressing the key when the stimulus was the long noise burst) is indicated for each point below the error bar. Each point represents approximately 100 responses to brief noise bursts and 100 "catch trials."

bursts by pressing a telegraph key as quickly as possible after the offset of the noise burst. Each trial was initiated by the $E$, who gave a verbal warning signal. One second after the verbal warning signal, a second warning signal occurred. It was a noise burst of $200 \mathrm{msec}$ duration. One second after the termination of the second warning signal, the critical stimulus was presented. It was either (with probability 0.5 ) a brief noise burst, to which a response was required, or (also with probability 0.5 ) a "catch trial," consisting of a 1-sec-long noise burst, to which no response was required. Since very few false responses (pressing the key when the stimulus was the long noise burst) occurred, it is clear that the $S$ was responding to stimulus offset and not to stimulus onset. RTs were measured to the nearest millisecond.

Trials were presented in blocks of 50 , during which the duration of the short noise burst (test stimulus) was kept fixed. In order to familiarize the $S$ with the test-stimulus duration, each block was preceded by four stimulus sequences, to which the $S$ was asked to listen without responding. The $S$ was told that the four preliminary sequences would contain a short noise burst, a long one. a short one, and a long one, in that order. The block of 50 trials followed immediately. Each block lasted approximately $7 \mathrm{~min}$.

The stimulus durations were $5,10,30,50,100,150$, and $200 \mathrm{msec}$. All seven stimulus durations were tested in each of four sessions for each $S$. The durations were presented in random arder in each session.

For each duration, in each session, the (approximately 25) RTs were averaged and the standard deviation computed. In order to eliminate aberrant data, which probably result from either anticipation or from momentary lapse of attention, the following computational procedure was used. Limits of the mean \pm 2 standard deviations were computed. If any RTs fell outside these limits, they were eliminated and the mean recomputed. This corrected mean was used in subsequent calculations.

\section{RESULTS}

The results for the two Ss are shown in Fig. 1. Each point represents the mean of the four corrected mean RTs obtained in four sessions. The error bars indicate \pm 1 standard error of the mean of the four corrected mean RTs. The number of false responses (pressing the key when the stimulus was the long noise burst) was small. being two or less for 8 of the 14 points and being five or less for all but 1 of the points.

For both Ss, the RT is essentially constant as a function of stimulus duration, except for the 100- and 150-msec durations. For the five durations excluding 100 and $150 \mathrm{msec}$, the mean RTs plotted in Fig. 1 for S G.N. are within $6 \mathrm{msec}$ of $209 \mathrm{msec}$; for S J.G., the mean RTs for these five durations are within $2 \mathrm{msec}$ of $185 \mathrm{msec}$.

For the 100- and 150-msec durations, a dip of 10-15 msec occurs in the mean RTs for both Ss. This result is seen to be more highly significant for $S J . G$., who exhibits less session-to-session variability than S G.N.; nevertheless, the dip in the mean RTs appears to be nearly identical for both Ss.

\section{DISCUSSION}

The finding of an essentially constant RT as a function of stimulus duration (excluding the 100- and $150-\mathrm{msec}$ durations) shows clearly that the delayed perception of stimulus offset obtained by Efron does not play a role in the task of responding to stimulus offset. That is, the time at which a response to stimulus offset can be initiated is independent of the time at which the offset is perceived, as determined by the simultaneity-judgment task of Efron.

The lack of correspondence between time of perception of offset and time of reaction to offset is not completely surprising, especially as the finding of constant RT is the common sense result if Efron's data on time of perception of offset are disregarded. Among the precedents for the result that RT does not always correspond to perceptual variables is an experiment of Fehrer and Raab (1962). They found that RT to a target was independent of whether the target appeared alone or whether its phenomenal brightness was reduced by metacontrast.

More directly related to the present experiment is an example of the lack of correspondence between time of perception of stimulus offset and performance in discrimination tasks involving stimulus offset. In the discrimination studies, the $S$ is required to discriminate between stimuli of different durations. Weber fractions ( $\triangle D / D$, where $D$ is the stimulus duration) have been obtained by Creelman (1961), Small and Campbell 
(1962), and Abel (1972), and are typically of the order of 0.1 to 0.5 for tone bursts and noise bursts whose durations are in the range of several milliseconds to several $100 \mathrm{msec}$. Clearly, it is possible to discriminate between two brief stimuli of different durations, even though the offsets of the stimuli would be perceived, according to the Efron determination, at a fixed interval of time after the respective stimulus onsets.

Another way of understanding the lack of correspondence between reaction time and time of perception is in terms of advantage to the S. Suppose, as seems quite likely, that more than one temporally distinct representation of stimulus offset occurs in the nervous system. It is clear that utilizing the earliest possible representation would be the best way to minimize $\mathrm{RT}$, as the $\mathrm{S}$ is instructed to do. In the Efron paradigm, on the other hand, the identification of perception of stimulus offset with the earliest neural representation thereof does not necessarily confer an advantage.

Finally, the result of a decrease in RT for the 100 and $150-\mathrm{msec}$ durations was entirely unexpected, and no simple explanation has yet suggested itself. It can, however, be noted that the anomalous decrease occurs in the region of Efron's critical duration of $130 \mathrm{msec}$.

\section{REFERENCES}

Abel, S. M. Duration discrimination of noise and tone bursts. Journal of the Acoustical Society of America, 1972, 51, 1219-1223.

Creelman, C. D. Human discrimination of auditory duration. Journal of the Acoustical Society of America, 1961, 34, 582-593.

Efron, R. The relationship between the duration of a stimulus and the duration of a perception. Neuropsychologia, 1970a, 8, 37-55.

Efron, R. The minimum duration of a perception. Neuropsychologia, 1970b, 8, 57-63.

Efron, R. Effect of stimulus duration on perceptual onset and offset latencies. Perception \& Psychophysics, 1970c, 8, 231-234.

Efron, R. An invariant characteristic of perceptual systems in the time domain. In S. Kornblum (Ed.), Attention and performance IV. New York: Academic Press, 1973.

Fehrer, E., \& Raab, D. Reaction time to stimuli masked by metacontrast. Journal of Experimental Psychology, 1962,63, 143-147.

Small, A. M., Jr., \& Campbell, R. A. Temporal differential sensitivity for auditory stimuli. American Journal of Psychology, 1962, 75, 401-410.

(Received for publication August 25, 1972; revision received December 4,1972 .) 